\title{
GW23-e2061 COMPARATIVE STUDY BETWEEN RIGHT VENTRICULAR OUTFLOW TRACT AND RIGHT VENTRICULAR APEX PACING ON CARDIAC FUNCTION
}

doi:10.1136/heartjnl-2012-302920m.4

Faquan Li, Wei Liao, Zhanglin Yan, Faquan Li. The First Affiliated Hospital of Gannan Medical College

Objectives To evaluate cardiac function change in patients using the right ventricular outflow tract and right ventricular apex pacing.

Methods 119 patients with bradyarrhythmias in our hospital were consecutive selected, cardiac pacemaker implantation are in line with class one indications. exclude, chronic atrial fibrillation, cardiac function three or four grade (NYHA), acute coronary syndrome and other serious medical diseases. And 60 patients randomly selected for right ventricular outflow tract pacing (DDD), (group A). 59 cases (group B), selected for right ventricular apex pacing in. Both groups were no significant difference with age, gender, type of arrhythmia, heart function.

Results The two groups according to the cumulative percent ventricular pacing (cum\% VP $>50 \%<100 \%$, 42 cases in group A, 38 cases in group $\mathrm{B}$, between the two groups was not statistically significant; but the left ventricular ejection fraction (LVEF) in group A higher than in group $\mathrm{B}$, and B-type natriuretic peptide (BNP) decreased in group $A$ compare with group $B(p<0.05)$. Group $A$ after 18 months compared with preoperative LVEF has a certain degree increased, BNP decreased ( $p>0.05$ ), group B after 18 months compared with preoperative LVEF slightly lower, with a certain degree of increased BNP ( $p>0.05)$.

Conclusions Right ventricular outflow tract pacing can improve heart function compared with right ventricular apex pacing, and to some extent improve heart function compared with preoperative too. 